\title{
COMPARING INNOVATIVE XR SYSTEMS IN CULTURAL HERITAGE. A CASE STUDY
}

\author{
M. Carrozzino ${ }^{1, *}$, G-D. Voinea ${ }^{2}$, M. Duguleană ${ }^{2}$, R. G. Boboc $^{2}$, M. Bergamasco $^{1}$ \\ ${ }^{1}$ TeCIP, Scuola Superiore di Studi Universitari e di Perfezionamento Sant'Anna, 56127 Pisa, Italy - (m.carrozzino, \\ m.bergamasco)@sssup.it \\ ${ }^{2}$ Faculty of Mechanical Engineering, Universitatea Transilvania din Braşov, 500036 Braşov, Romania - (daniel.voinea, \\ mihai.duguleana, razvan.boboc)@unitbv.ro
}

KEY WORDS: User Study, Virtual Reality, Augmented Reality, Mixed Reality, Education, Interaction, Manipulability

\begin{abstract}
:
The technological advances made in the recent years in the field of Information and Communication Technologies (ICT) have led to a revolution in many adjacent areas. One of these is Cultural Heritage $(\mathrm{CH})$, as the new array of XR technologies (a concept integrating Augmented, Mixed and Virtual Reality) is offering new interaction possibilities. The purpose of this paper is to compare several such technologies at application level, and to classify them based on common features such as Interaction, Manipulability, Ease of Use and others. The study presented in this paper was carried out during the H2020 project eHERITAGE ('Expanding the Research and Innovation Capacity in Cultural Heritage Virtual Reality Applications'). The action had as output multiple XR systems/applications. We compare 4 of them: a smartphone-based AR application, a digital book with 3D elements deployed on a large touchscreen, a large holographic display and a bow simulator built using a VR headset and a mechatronic system. We evaluate these innovative systems in the context of the 2 educational events, when over 4000 participants interacted with our team. Results show that systems with greater interaction and immersion features were preferred over the others. Furthermore, Mixed Reality was preferred over Virtual and Augmented Reality.
\end{abstract}

\section{INTRODUCTION}

\subsection{State of the Art}

Cultural heritage $(\mathrm{CH})$ is going through a technological transformation that has a positive impact on all its stakeholders. Advances in Information and Communication Technologies (ICT) offer $\mathrm{CH}$ new interaction possibilities. Starting with small smart watch screens and ending with immersive head-mounted displays, mixed reality solutions enhance users' experience, as visual output still has a lot of room for improvement. A comprehensive state of the art on visual interfaces used in $\mathrm{CH}$ can be found in (De Carolis, 2018). Is however a good visual experience the only factor contributing to a successful experience? This study aims to investigate this by comparing several innovative technologies/systems deployed in various applications in $\mathrm{CH}$. We use a questionnaire to evaluate how education, arts and entertainment sectors can beneficiate by the increased visitor satisfaction offered by these systems, and to find out which one is perceived as the best technology/system/application.

Up to this moment, there are just a few innovative technologies that paved their way into the $\mathrm{CH}$ industry. Virtual museum systems e.g. adopt a playful and educational approach, which is an effective solution in communicating cultural contents (Barbieri, 2017). Augmented Reality (AR) mobile applications are a cheap alternative to virtual reality setups. (Sannikov, 2015) and in (Pallud, 2017) present the importance of AR and $3 \mathrm{D}$ visualization technologies in creating interactive educational content. Other dedicated systems specifically built to be used in science festivals are also making a huge progress (Tom Dieck, 2018).
These new technologies such as AR and VR, create bridges of interaction between us and the environment around, and this mixture between real and virtual worlds is often referred to as "X-Reality" or "XR" (Mann, 2018). The term shows the vastness of the spectrum of technologies that are becoming available (Wallgrün, 2018).

VR has been widely used for the preservation and promotion of $\mathrm{CH}$ for people who are not able to physically visit museums (Loizides, 2014). Some studies have assessed the impact of VR technologies, showing that they exert a strong influence on tourism (Wei, 2019). Speaking of tourism, AR is also one of the emerging technologies used in most travelled sites (Lee, 2015). For instance, mobile augmented reality (MAR) provides a lot of opportunities for visitors to have engaging experiences (Galatis, 2016), (Angelopoulou, 2012). The relationship between innovation and CH should be increasingly closer (Garau, 2017). The experience of visiting a museum could be enriched using wearable AR displays (Vainstein, 2016), but there are still few studies that explore the potential of these technologies in relationship with the user's engagement. Aitamurto (2018) presents a guide on the positive impact that AR has on users' emotional and intellectual connections to the art. He (2018) presents another study in which he examins the impact of AR design elements on visitor's tourism experience and willingness to pay more.

Head-mounted display (HMD) are portable immersive systems offering promising opportunities to improve the user experience and interaction in $\mathrm{CH}$ field (Ferrari, 2017). Ioannides (2016) developed an interactive book providing an immersive digital heritage experience.

\footnotetext{
* Corresponding author
} 
Although there are a lot of approaches and implementations, there is generally a lack of knowledge regarding the technology acceptance (Chuach, 2018), as well as the evaluation of the optimal solution for a particular case from the multitude of existing systems. This study aims to offer a deep perspective on the difference between various technologies and their possible applications.

\subsection{EHERITAGE project}

The project eHERITAGE is a Coordination and Support initiative, which had three main objectives: to increase scope and competitive research, to enhance the training and mobility of researchers, and to continuously extend the research and innovation capacity of the coordinator, the Transylvania University of Brasov.

We achieved this desiderates in several ways. One of the most important actions taken was to collaborate with local, national and international entities related to the field of study. Another strong point of the project is the eHERITAGE had the opportunity to reach maturity in 2018, during the European Year of Cultural Heritage. Given this turn of events, eHERITAGE managed to influence social, economic and cultural environments at national and international levels, by participating in numerous events and by establishing new strategic partnerships with other research institutes or academic entities.

One of the side activities of the project was to build several VR systems showcased during various events, such as: the EU Open Day (2018) in Brussels, Belgium; European Researchers' Night (2017 and 2018) in Braşov, Romania; Creative Summer School (2016-2018) in Constanța, Romania; LuBeC - Lucca Beni Culturali (2017) in Lucca, Italy; Internet Festival (2017) in Pisa, Italy; TEDx Constanța (2017) in Constanța, Romania; VRTCH'18 (2018) in Braşov, Romania; ICIF (2018) in Shenzen, China; GFCO (2018) in Brașov, Romania.

Thousands of people had the chance to test equipment running virtual heritage scenarios at these trade shows and expositions. We estimate that throughout the lifespan of the project, more than 20.000 people have discovered our work in cultural heritage. It was during this time that we discovered some of the systems/applications are more appreciated than others, a concept which led to this study.

\subsection{Events}

This study was carried out during 2 events from 2018: "Graduates in Front of Companies" (GFCO) and Researcher's Night (RN). GFCO is an annual event hosted by the Transylvania University of Brasov, one which brings together academia and companies. Hundreds of students have to chance to showcase their projects to CEOs and representatives from human resources from top companies based in Brasov. Our team presented the systems as intellectual outputs produced by the university, available for testing by any student or company representative. We estimate that around 100 people interacted with our products during GFCO 2018, mostly students, PhD candidates, university staff and employees of the companies present at this events and working in similar fields of study such as ICT or cultural heritage.

The target group of GFCO is, however, rather small, when compared to the RN. RN is another annual event whose aim is to popularize science and to promote fun learning. $\mathrm{RN}$ is an event that tries to raise public awareness on the role of research in the society of the $21^{\text {st }}$ century. The initiative is centered on young people with general interest in technology. The idea behind this event is that visitors can find out more about the research activities around their place of living, and how these positively impact people's regular daily lives. During the RN 2018, eHERITAGE had an approximate audience of 4000 participants, spread across 2 days. We conducted hands-on experiments, equipment presentations, and group debates related to the equipment showcased here (see Figure 1).

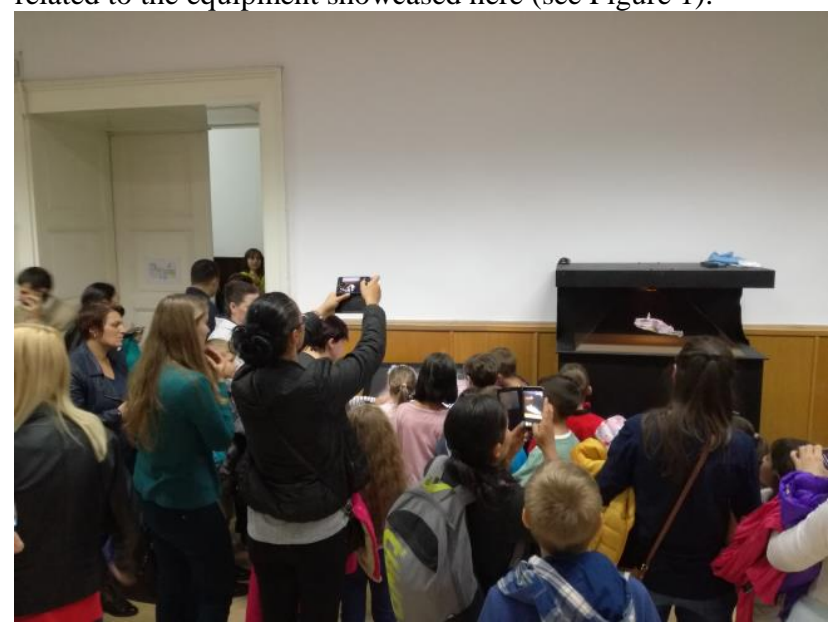

Figure 1. Users testing various systems during Researchers' Night 2018

Combined, the participants from these two events provided enough data to work on the proposed study.

\section{SYSTEMS AND APPLICATIONS}

In order to reach a common ground and to better understand the concept of comparing different technologies and systems, we are presenting bellow in detail the four systems which we implemented and which want to evaluate.

\subsection{Mobile AR exposition}

MAR is today a well-established technology. This was made possible by the technological advances in the processor, memory, screen and camera of the smartphones. One of the most famous applications used by millions of people is e.g. Pokemon GO. Although this game had only limited AR features, it had a huge success. People were attracted by the novelty of the technology and by the established characters. However, the application needed to be compatible with a wide array of devices, and thus the implementation of the $\mathrm{AR}$ scenarios was rather symbolic (Althoff, 2016).

Since the launch of Pokemon GO, things have evolved rapidly. A new technology usingcustom AR hardware is the Google Tango project. Based on the position, orientation and movement obtained from the accelerometers and gyroscopes of compatible smartphones, the platform is able to integrate three types of functionalities: motion-tracking (using visual cues from the working environment) environment, area learning and depth perception. Google Tango devices are able to detect distances, sizes, and surfaces working in the environment.

Although revolutionary, Google Tango was abandoned for a much cheaper technology, ARCore, which does not require special cameras and relies on devices normally equipped inside mobile devices, and thus it is suitable for any kind of smartphone. The most similar sibling of the ARCore is the ARToolkit, available for iOS platforms. Together, these two libraries represent most the current state-of-the art in MAR. 
Although versatile, ARCore/ARToolkit have inferior capabilities when compared with a Google Tango device such as the Lenovo Phab 2 Pro, the one used in our presentations (see Figure 2).

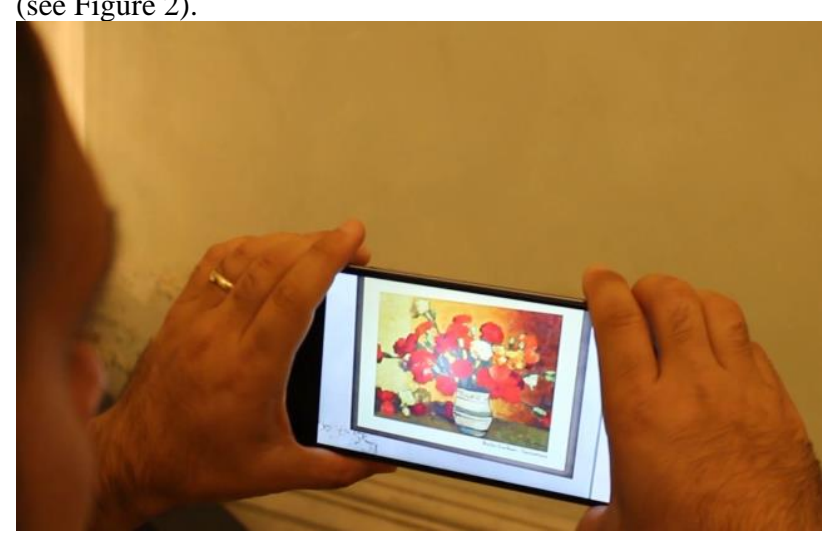

Figure 2. User looking at the painting "Still Nature -

Carnations" by Romanian painter Stefan Luchian

The scenario presented to the visitors from GFCO and RN was based on some of the most interesting paintings of famous Romanian painters such as Stefan Luchian, Adrian Ghenie and Nicolae Grigorescu. Several of their works were virtually placed on the free walls of the exhibition space. This was made possible thanks to a pre-calibration and area learning procedure. After all the paintings were set at custom coordinates, users could use two Lenovo Phab 2 Pro phones to wander around and inspect the virtual exposition. Each painting had a label next to it, presenting the name of the work, the artist and the year of making.

\subsection{VR Book}

Another interesting setup able to attract and engage the public was a VR book jointly developed during eHERITAGE, by the Transylvania University of Brasov (Romania) and Scuola Superiore Sant'Anna di Pisa (Italy). This innovative and easily replicable system proposes a digital book enhanced with 3D objects and information cues. Our aim was to capitalize a vestige sitting in the warehouse of the History Museum in Brasov. This is the Constitutio Criminalis Theresiana (also known as Nemesis Theresiana or only Theresiana), a criminal code drafted in Austria during the reign of Maria Theresa, Queen of Hungary.

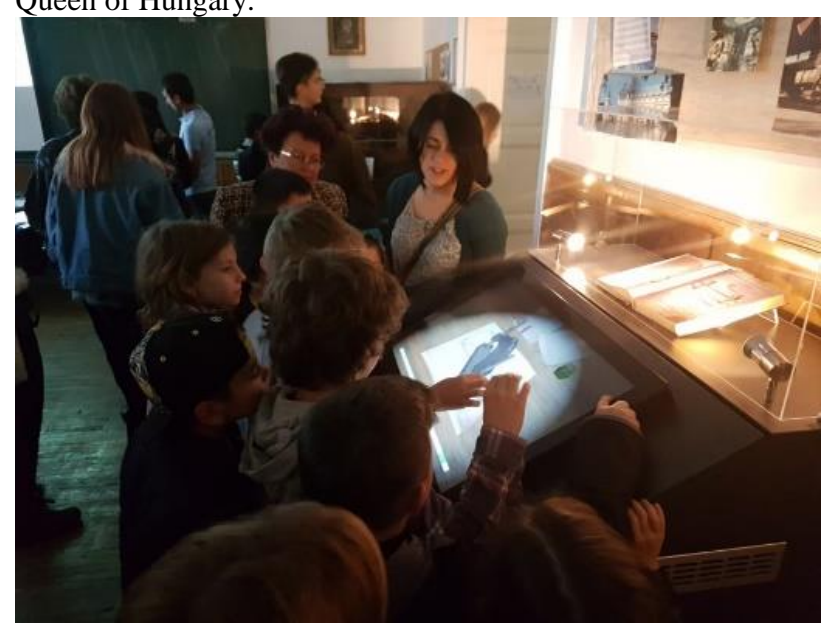

Figure 3. Researchers' Night participants using the digital book

The book was drafted in Vienna in 1768 and ensured the uniform application of Criminal Law in Austria and Bohemia.
Theresiana aimed to reduce the torture that was applied at the time to strictly regulate the methods of torture. Using a large tactile display, we have built a museum-ready stand, as presented in Figure 3. Various torture instruments have been modelled and animated. Multiple sections of the book were translated in English and Romanian and placed as information bullets throughout the contents of the manuscript. Navigation was also enhanced thanks to a user interface enabling fast page turning forwards and backwards.

\subsection{Holographic Stand}

The holographic stand presented in Figure 4 is a system built by the team from Transylvania University of Brasov in the last year of the project. The stand showcases several fortified churches from "Țara Bâsei", a small region of Transylvania, Romania. Although some of these churches are on UNESCO's list of protected monuments, they are not usually included in mainstream visiting circuits. Our aim was to increase the visibility of these landmarks for tourists who usually only reach the most commercial historic landmarks.

We have used photogrammetry to build the 3D models of 6 fortified churches and of the Castle Bran. Tens of thousands of pictures were processed to obtain optimized models of these edifices. Some of these models are available in the Repository section from the project's website (eHERITAGE Project, 2018). A Bluetooth connected tablet allows users to select among the models of the churches while also providing additional information for each selected item, such as the founding year, important historical aspects, as well as current challenges and future conservation and management plans.

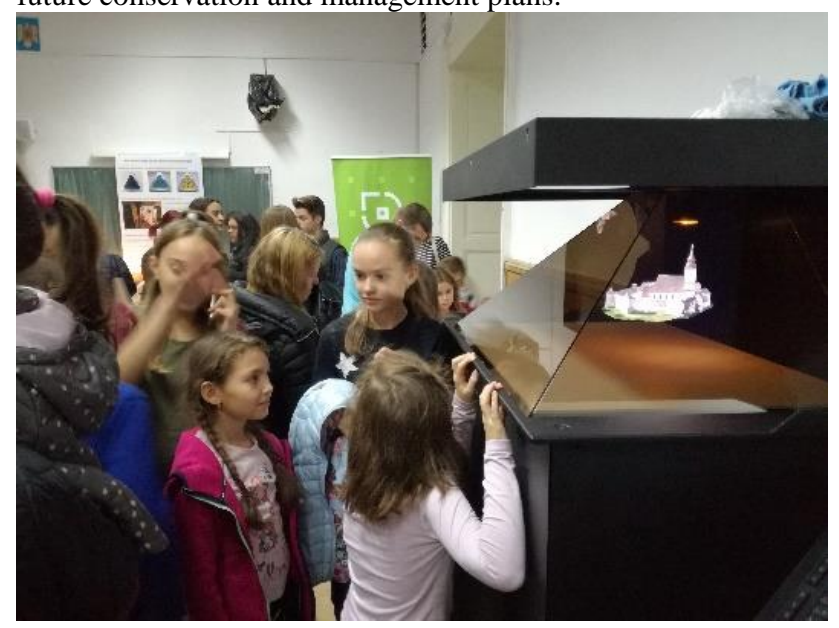

Figure 4. Researchers' Night participants looking at the holographic display

\subsection{Bow simulator}

Another innovative system designed by eHERITAGE staff is the haptic bow. We've built an immersive virtual reality simulator for recreating the experience of shooting with various types of old bows, based on a customized haptic interface.

The research focused on optimizing the shooting experience by using the force characteristic measured from real replicas, as well as handling other important archery features such as the length of the draw or the weight of the bow.

This highly interactive equipment allows users not only to experience an immersive archery scenario, but also to customize the force characteristic of the bow they want to shoot with, 
making a difference between i.e. English longbows and short recurved Turkish horse bows.

The VR scenario takes place at the base of the famous Bran Castle. Users can shoot arrows at 5 scarecrows and a large circular target. The scene is built in Unity, while the model of the Bran Castle is built through photogrammetry. The application can be run with any of the commercial VR headsets available today. During these two events, we used Oculus Rift (see Figure 5).

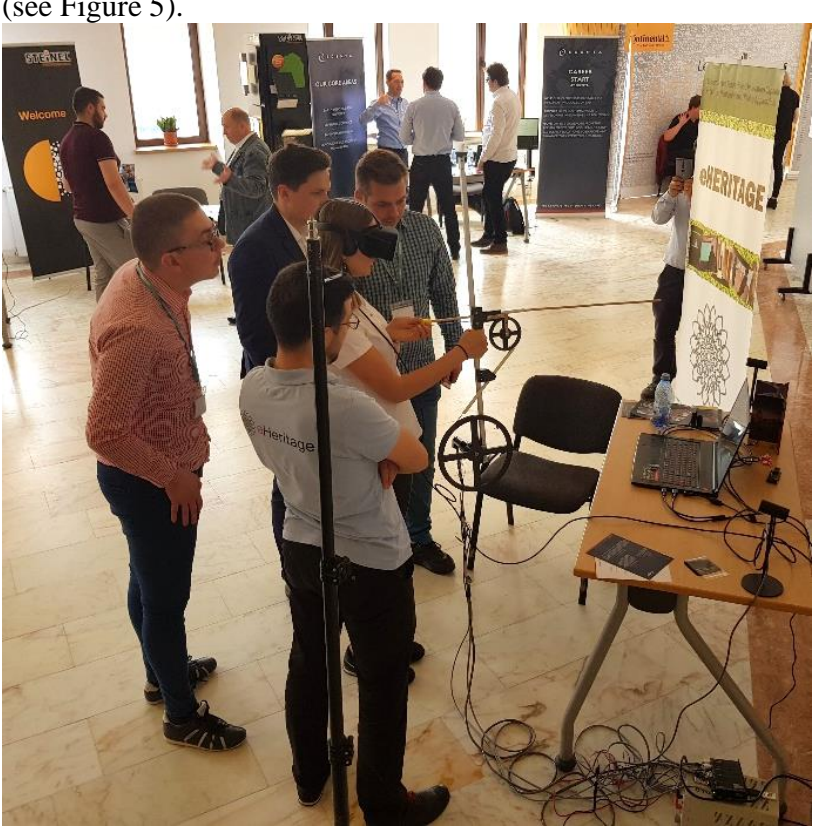

Figure 5. Students trying the haptic bow at GFCO

\section{EVALUATION}

Given the large number of attendants to the two events presented above, we used the naturalistic observation method to record the behaviour of individuals that interacted with the systems. We present below the methodology and the results of the study.

\subsection{Methodology}

3.1.1 Participants: The observation took place in three different days: on the 24th of April, 2018 (at GFCO) and on the 28th and the 29th of September, 2018 (at RN). The events described in the previous section attracted people with particular interest in technology, such as young children, college students, parents and tutors.

Out of the people who got in contact with our stands and our team, we managed to record a total of 126 participants, which tested various pieces of equipment. We observed 47 females and 79 males. The majority seemed to be young children and adults ranging from 12 to 65 years. Some of the subjects tested all equipment while others only tested 1 or 2 installations. This is a result not only of the attractiveness of the devices, but also of circumstantial factors such as the size of the ques, the time needed to actually test the installation, the temporal crowding of the exposition space or the physical occlusion which occasionally happens in this type of events, the availability of the observer or the perceived reward.

In Figure 6 we present the number of trials distributed to each tested equipment. The percent values were obtained by dividing the number of tests for each piece of equipment with the sum of the total number of tests. The VR book and the holographic stand were the most frequented installations due to the fact that they were also the most physically accessible. The smartphones with mobile AR were the least used, mainly because they required 1-on-1 tutoring before testing.

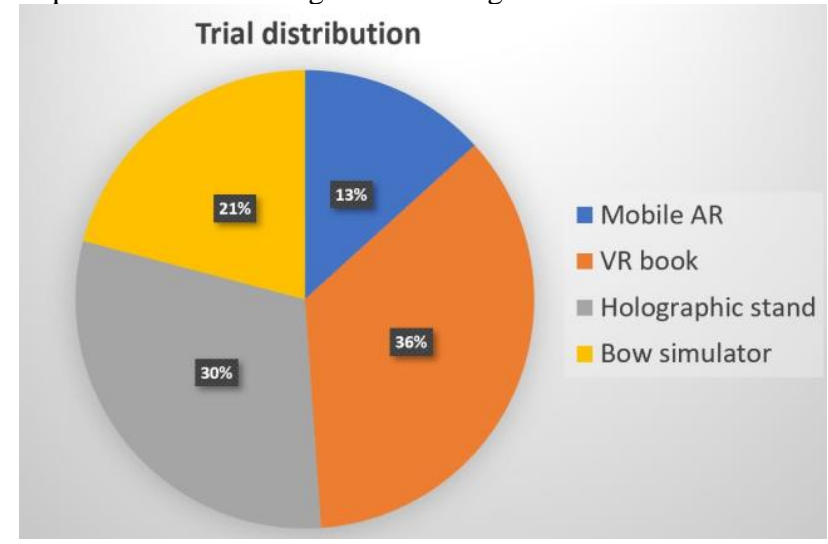

Figure 6. Equipment usage

3.1.2 Parameters: The main parameters which we pursued are Interaction (features related to the user interface, the way subjects deal with the application scenarios), Manipulability (features related to ergonomics, how tiering or how comfortable is to use the equipment), Ease of use (whether the person requires special training or advice before using the device or system), Ease of learning (if, when and how users become more certain on using the proposed systems), Novelty (assessing the degree of novelty, the way people react to new experiences) and Social influence (social behaviour related to the equipment, including recommendations, social media interactions, group discussions and so on). We rated these factors on a scale from 1 to 5,1 being the least favourable and 5 the most favourable grade. Furthermore, we split the resulted averages of these ratings into 3 intervals for easier understanding and reporting: Low (between 1-2.33), Medium (between 2.33-3.66) and High (between 3.66-5).

3.1.3 Procedure: The proposed parameters were extracted using natural observation, a data collection method which allows researchers to note behaviour and activities without interfering with the subjects. The users were visually selected as soon as they interacted with one of our team members or with one of our systems, during the 2 events described in the previous sections. When observers noticed the subject is no longer paying attention to the proposed stands/apps, they moved to the next available one. The data observed was recorded on datasheets, containing objective information such as gender, and well as subjective information (approximative age and rating the study parameters). Sometimes, subjects provided feedback on the installations, either technical or historical, oriented on the content of the systems. We also noted these down, in an attempt to quantify the study parameters of each individual as close as possible to their real value.

3.1.4 Observer reliability: The observers are members of the eHERITAGE project. They were instructed during a project meeting on how to collect the data and rate users, without actually interfering with their activities. The certain guidelines referring to the naturalist observation and other means of recording social cues from our environment were presented to the team involved in this line of work and consequently reinforced in the beginning of the 2 events: GFCO and RN. 


\begin{tabular}{|l|l|l|l|l|l|l|}
\hline System & Interaction & Manipulability & Ease of use & Ease of learning & Novelty & Social influence \\
\hline Mobile AR & Low & High & Medium & High & Medium & High \\
VR book & Medium & n/a & High & High & Medium & Medium \\
Holographic stand & Medium & n/a & High & High & High & Low \\
Bow simulator & High & Medium & Low & Medium & High & High \\
\hline
\end{tabular}

Table 1. Study parameters for each piece of equipment

\subsection{Results}

The results of this study are presented in Table 1. Computed averages show the strong points and the weaknesses of each of the 4 systems.

The MAR setup has high ratings in Manipulability (it's easy to carry around), Ease of leaning (after getting in contact with an AR demo, subjects understood precisely what to expect from this technology and how to use it) and Social Influence (users can interact with others easily when using this system, and moreover, they can capture the screen of the application and share it on the social channels). The drawbacks are the Ease of Use (which is medium based on the ratings), the Novelty (many have already heard and tested AR apps, thus resulting another medium rating) and the Interaction parameters (diminished possibility to physically interact with the small screen of a smartphone or tablet).

The digital book with 3D elements is implemented on a large touch screen mounted on a fixed museum-ready stand. Since the interaction involves only using the touch screen, users found the system to be easy to use and easy to learn. Although filled with a large content, the system cannot be rated for Manipulability (users only make swipe gestures to browse the book). The interaction resulted to be medium on average. The historic content translates into a large amount of data, all having a certain degree of novelty and social influence.

The holographic stand has high levels of the following parameters: Ease of use, Ease of leaning and Novelty. Although the concept behind a hologram is relatively old, almost all participants were surprised by this technology. The tablet linked to the stand enabling users to select their desired 3D model provides medium levels of interaction. Unfortunately, the Social influence is reduced, most likely because of the dimensions of the stand.

The haptic bow is trivially the most interactive system created by us, and thus, the most appealing. There was always a crowd forming, queuing to shoot with the archery simulator. Besides the interaction feature, the novelty and the social influence are also rated 'High'. The Ease of Use and the Manipulability are the biggest setbacks of this system, and they are most likely caused by the physical nature of the system, which needs to be installed manually on each user.

\section{CONCLUSIONS}

Overall, the haptic bow simulator was the main attraction at both events, as it incorporates high levels of interaction and social influence, which in turn increase the perceived presence and thus the appeal of the users. This novel application can be used to learn archery or just for entertainment purposes. Nevertheless, all the equipment was well received by the audience, from young children to adults, thanks to the novelty parameter, which was either 'Medium' or 'High' in all 4 cases. A good future development which is worth pursuing is quantifying the importance of each of the study parameters - it seems that Interaction and Manipulability play a role bigger than the rest of the other parameters.

\section{ACKNOWLEDGEMENTS}

This paper is supported by European Union's Horizon 2020 research and innovation programme under grant agreement No 692103, project eHERITAGE (Expanding the Research and Innovation Capacity in Cultural Heritage Virtual Reality Applications).

\section{REFERENCES}

Barbieri, L., Bruno, F., \& Muzzupappa, M. (2017). Virtual museum system evaluation through user studies. Journal of Cultural Heritage, 26, 101-108.

De Carolis, B. N., Gena, C., Kuflik, T., Origlia, A., \& Raptis, G. E. (2018, May). AVI-CH 2018: Advanced Visual Interfaces for Cultural Heritage. In Proceedings of the 2018 International Conference on Advanced Visual Interfaces (p. 6). ACM.

Pallud, J. (2017). Impact of interactive technologies on stimulating learning experiences in a museum. Information \& Management, 54(4), 465-478.

Sannikov, S., Zhdanov, F., Chebotarev, P., \& Rabinovich, P. (2015). Interactive educational content based on augmented reality and 3D visualization. Procedia Computer Science, 66, 720-729.

Tom Dieck, M. C., Jung, T. H., \& Rauschnabel, P. A. (2018). Determining visitor engagement through augmented reality at science festivals: An experience economy perspective. Computers in Human Behavior, 82, 44-53.

Mann, S., Furness, T., Yuan, Y., Iorio, J., \& Wang, Z. (2018). All Reality: Virtual, Augmented, Mixed (X), Mediated (X,Y), and Multimediated Reality. arXiv preprint.

Wallgrün, J.O., Huang, J., Zhao, J., Ebert, C., Roddy, P., Awe, J., Murtha, T., \& Klippel, A. (2018). Immersive Technologies and Experiences for Archaeological Site Exploration and Analysis. Lecture Notes in Geoinformation and Cartography, 198809, pp. 307-314.

Loizides, F., El Kater, A., Terlikas, C., Lanitis, A., \& Michael, D. (2014). Presenting Cypriot Cultural Heritage in Virtual Reality: A User Evaluation. In EuroMed 2014: Digital Heritage. Progress in Cultural Heritage: Documentation, Preservation, and Protection, Springer International Publishing, pp. 572-579.

Wei, W., Qi, R., \& Zhang, L. (2019). Effects of virtual reality on theme park visitors' experience and behaviors: A presence perspective', Tourism Management, 71, pp. 282-293.

Lee, H., Chung, N., \& Jung, T. (2015). Examining the Cultural Differences in Acceptance of Mobile Augmented Reality: Comparison of South Korea and Ireland. In Information and 
Communication Technologies in Tourism, Springer International Publishing, pp. 477-491.

Galatis, P., Gavalas, D., Kasapakis, V., Pantziou, G., \& Zaroliagis, C. (2016) Mobile Augmented Reality Guides in Cultural Heritage. In Proceedings of the 8th EAI International Conference on Mobile Computing, Applications and Services.

Angelopoulou, A., Economou, D., Bouki, V., Psarrou, A., Jin, L., Pritchard, C., \& Kolyda, F. (2012). Mobile Augmented Reality for Cultural Heritage. In MOBILWARE 2011: Mobile Wireless Middleware, Operating Systems, and Applications, pp. $15-22$.

Garau, C. (2017). Emerging Technologies and Cultural Tourism: Opportunities for a Cultural Urban Tourism Research Agenda. In Bellini, N., and Pasquinelli, C. (Eds.): Tourism in the City : Towards an Integrative Agenda on Urban Tourism, Springer International Publishing, pp. 67-80.

Vainstein, N., Kuflik, T., \& Lanir, J. (2016) Towards Using Mobile, Head-Worn Displays in Cultural Heritage: User Requirements and a Research Agenda. In Proceedings of the 21st International Conference on Intelligent User Interfaces, Sonoma, California, pp. 327-331.

Aitamurto, T., Boin, J.-B., Chen, K., Cherif, A., \& Shridhar, S. (2018). The Impact of Augmented Reality on Art Engagement: Liking, Impression of Learning, and Distraction. In VAMR 2018: Virtual, Augmented and Mixed Reality: Applications in Health, Cultural Heritage, and Industry, Springer International Publishing, pp. 153-171.

He, Z., Wu, L., \& Li, X. (2018). When art meets tech: The role of augmented reality in enhancing museum experiences and purchase intentions. Tourism Management, 68, pp. 127-139.

Ferrari, F., \& Medici, M. (2017). The Virtual Experience for Cultural Heritage: Methods and Tools Comparison for Geguti Palace in Kutaisi, Georgia. Proceedings, 1(9).

Ioannides, M., Athanasiou, V., Chatzigrigoriou, P., Papageorgiou, E., Leventis, G., Nikolakopoulou, V., \& Sovis, C. (2016). Immersive Digital Heritage Experience with the Use of Interactive Technology. In EuroMed 2016: Digital Heritage. Progress in Cultural Heritage: Documentation, Preservation, and Protection, Springer International Publishing, pp. 265-271.

Chuah, S. (2018) Why and Who Will Adopt Extended Reality Technology? Literature Review, Synthesis, and Future Research Agenda. Universiti Sains Malaysia, School of Management: Kuala Lumpur, Malaysia.

Althoff, T., White, R. W., \& Horvitz, E. (2016). Influence of Pokémon Go on physical activity: study and implications. Journal of medical Internet research, 18 (12).

eHERITAGE Project, 2018. Available at eheritage.org (12 March 2019). 